\title{
Characterizing the Impact of Multi-frequency and Multi-power on Localization Accuracy
}

\author{
Xiuyuan Zheng, Hongbo Liu \\ Jie Yang, Yingying Chen \\ Department of ECE \\ Stevens Institute of Technology \\ Hoboken, NJ 07030 \\ \{xzheng1, hliu3, jyang, yingying.chen\}@ stevens.edu
}

\author{
John-Austen Francisco \\ Richard P. Martin \\ Department of CS \\ Rutgers University \\ Piscataway, NJ 08854 \\ \{deymious, rmartin\}@cs.rutgers.edu
}

\author{
Xiaoyan Li \\ Department of CS \\ Lafayette College \\ Easton, PA 18042 \\ lix@lafayette.edu
}

\begin{abstract}
Wireless localization using the received signal strength (RSS) can have tremendous savings over using specialized positioning infrastructures. In this work, we explore improving RSS localization performance in multipath environments by varying the transmitter's signal power and frequency. Using a theoretical analysis, we first show how selection of different signal powers and frequencies can improve localization accuracy for the least squares algorithm. We next develop a set of selection methods that attempt to select the combinations of power and frequencies which minimize the localization error. Our selection methods are based on the observed standard deviations of RSS as well as algorithm specific residuals. Using active RFID tags, we experimentally characterize the effect of using multiple signal powers and frequencies on a wide spectrum of RSS-based algorithms. We found that the performance of all the algorithms improves when leveraging on multiple power levels and frequencies, although different algorithms present different sensitivity in terms of localization accuracy under different selection methods.
\end{abstract}

\section{INTRODUCTION}

Using wireless devices to provide location is an emerging area that will impact a diverse set of applications including those in asset tracking, workflow management, geographic routing, and physical security. Given that wireless devices are carried by many people and objects, and all modern radio chipsets include the hardware necessary to measure the received signal strength (RSS) of transmitted packets, there is a tremendous cost and deployment advantage to re-using the existing RSS infrastructure of the communication network for localization.

The basic strategy of most RSS-based localization follows a typical pattern: a transmitter or receiver, called the device, measures the RSS to a number of landmarks with known positions. The resulting collection of RSS values, or fingerprint, is then used to position the device. A wide range of algorithmic strategies have been tried, ranging from minimization of least squares methods to machine learning approaches.

However, multipath effects are key challenge to wireless localization using RSS. These effects include shad- owing, i.e., blocking a signal, reflection, i.e., waves bouncing off an object, diffraction, i.e., waves spreading in response to obstacles, and refraction, i.e., waves bending as they pass through different mediums. These effects impact the RSS, and properly accounting for all of them in complex indoor environments is a difficult task. Typically, the average localization errors are around $10 \mathrm{ft}$ and maximum errors are often $30 \mathrm{ft}$ or more.

In this paper we explore one approach of improving RSS localization performance in face of multipath effects. Our strategy is for the transmitter to vary its signal frequency and power level, thus generating multiple RSS values between the device and each landmark. The resulting distinct RSS values for each frequency and power level combination create a larger fingerprint than is typical with a single frequency and power level. We call each frequency and power level combination a dimension of the fingerprint. We then apply a selection algorithm that attempts to create a fingerprint that minimizes the localization error.

In order to form a selection strategy, we must first define what a higher-quality fingerprint is. We use two metrics to help select the higher-quality fingerprint: the deviation of RSS readings and the residual. We further develop three selection strategies: (1) Whole Dimension, whereby all dimensions of frequency and power level are used for localization; (2) Matching Characteristic, whereby a fingerprint is constructed with all landmarks using the same frequency and power level combination; and (3) Constrained Landmark, whereby all landmarks are enforced to be used, but allowing any dimension to be chosen for a landmark.

We applied both a theoretical analysis as well as experimental validation of our approach. Our analysis centers on the Linear Least Squares (LLS) algorithm; it shows that selection should improve performance, and the selection should take the dimensions with the minimum variance within a dimension. We also experimentally applied our approach to a broad class of localization algorithms with a range of mathematical foundations, from lateration based, to fingerprint matching, to proba- 
bilistic based, and Bayesian Networks. Leveraging a real localization testbed, we programmed RFID transmitters with varying frequency and power level. We were able to vary the frequency at two levels, $400 \mathrm{MHz}$ and $900 \mathrm{MHz}$ as well as simultaneously vary the power by 3 levels, 10 , 5 and $0 \mathrm{dBm}$, resulting in 6 additional dimensions of RSS between a device and a landmark. We then used a tracedriven approach to evaluate the impact of our selection strategies on this broad class of localization algorithms.

We found that using multiple frequencies and power levels improved the performance of all the algorithms. An interesting effect common across all the algorithms is that median errors were substantially reduced. Examining a cumulative distribution function (CDF) of localization errors, the median errors improved over $40 \%$ for all the algorithms using multiple frequencies and power levels, whereas the improvement of $90^{\text {th }}$ percentile errors varied from $32 \%$ to $58 \%$ depending on the algorithm. We further found that different algorithms react differently to the selection strategies. Selection was necessary to improve performance and reduce computational cost compared to using Whole Dimension selection, although each algorithm required a slightly different selection strategy.

The remainder of our paper is organized as follows. We first provide background and related work in section II. Section III provides our selection metrics and describes the broad class of localization algorithms. We also present our theoretical analysis of linear least squares in Section III. Next, we describe our experimental methodology and present our selection strategies in Section IV. Section V evaluates the effectiveness of the various selection strategies on localization algorithms. Finally, Section VI concludes our work.

\section{RELATED WORK}

Localization has been researched in several settings: outdoor localization, indoor localization, and localization with the use of wireless sensor networks. In the indoor localization category, a wide range of technologies have been explored: ultrasound [11], infrared [13], 802.11, and custom radios. Within this wide variance, the works using 802.11 and signal strength [1], [6], [9] are most closely related to ours. Our work, however, does not propose any specific localization algorithm. Instead, we experiment to characterize the effect on a wide spectrum of RSS-based indoor localization algorithms when the wireless devices are equipped with multi-frequency and multi-power.

Due to properties of wireless signal propagation, both power level and frequency may inherently affect the performance of localization: The power level at which the signal is sent out decides how far the signal can travel; the frequency at which the signal is transmitted dictates how its propagation will be affected by the environment. Thus, many works have proposed to utilize them in different aspects of localization and position verification. [8] proposed to use multi-frequency to allow more accurate range estimation of RFID tags which is then used in localization [17]. [12] recognized the challenge for RSS-based localization if nodes in wireless sensor networks conduct power scheduling for topology control, then proposed to incorporate the power information into MAC layer message in order to correctly localize with such dynamic transmission power change. [15] proposed to have the anchor node information broadcasted and forwarded using different power levels so that hop count can provide better estimate for distance. Transmitting in multiple power levels is also proposed in [19] to provide rough area localization for underwater sensors. Finally, [18] proposed to use transmission power adjustment to verify location claims from new devices.

Comparing to the above, our work does not utilize the frequency or transmission power information directly. We focus on examining the effect on localization algorithms when multiple sets of RSS data (via different frequency and power level) can be selected and used in the system.

\section{Localization Algorithms And TheoretiC ANALYSIS}

In this section, we first define two selection metrics, the deviation and the residual, which are used to help select higher-quality RSS fingerprints. We then describe a broad class of localization algorithms using RSS, ranging from lateration based, to fingerprint matching, to probabilistic based, and to Bayesian Networks. For the linear least squares method, we provide a theoretical analysis to show how our selection metrics can improve the localization accuracy.

\section{A. Terminology}

We use the following definitions and terms to introduce the localization algorithms and selection metrics. The fingerprints (i.e., RSS readings) are generated at multiple locations. For a targeting device at location $j$, the fingerprint is represented as a vector $\left(\left(x_{j}, y_{j}\right), S_{j}\right)$ with $\left(x_{j}, y_{j}\right)$ as the location $j$ where the RSS vector is collected. For example, $S_{j}=\left(s_{1 j}^{L_{1}}, \ldots, s_{1 j}^{L_{q}}, \ldots, s_{i j}^{L_{1}}, \ldots, s_{i j}^{L_{q}} \ldots, s_{n j}^{L_{1}}, \ldots, s_{n j}^{L_{q}}\right)$ represent the RSS vector from $n$ landmarks with $L_{q}$ dimensions: element $s_{i j}^{L_{p}}$ represents the RSS reading of a targeting device at location $j$ from the $i$ th $(i=1 \ldots n)$ landmark on the $L_{p}$ th $\left(L_{p}=L_{1} \ldots L_{q}\right)$ dimension. In this work, a dimension represents each frequency and power level combination at a landmark. We note that certain localization algorithms need training data to build signal maps during the offline phase, which is used for the online real-time localization. We use the fingerprints collected from multiple locations to form the training data and denote $T$ as the training set with multiple vectors $\left(\left(x_{j}, y_{j}\right), S_{j}\right)$ for $j=1 \ldots m$, and $m$ is the number of locations used for training. 


\section{B. Selection Metrics}

Choosing the best fingerprint among the many possible combinations requires a characterization of the input RSS values. In this section we describe the two metrics we used for selection; the deviation of RSS readings and residual. A given combination of RSS values can be evaluated according to these metrics, and the best one is selected as input to the localization algorithm.

Deviation of RSS Readings. We define the deviation of RSS readings as the measurement of the Root Mean Square Error (RMSE) when fitting the RSS readings to the signal propagation model for each individual dimension under one landmark. Thus, for a targeting device at location $j$ in the $L_{p}$ th dimension of landmark $i$, the deviation of RSS readings can be represented as the RMSE of the estimated RSS $\hat{s}_{i j}^{L_{p}}$ from the fitted signal propagation model with respect to the observed RSS $s_{i j}^{L_{p}}$ :

$$
\operatorname{RMSE}\left(\hat{S}_{i}^{L_{p}}\right)=\sqrt{\frac{1}{m} \sum_{j=1}^{m}\left(\hat{s}_{i j}^{L_{p}}-s_{i j}^{L_{p}}\right)^{2}},
$$

where there are $m$ fingerprints, and $\hat{s}_{i j}^{L_{p}}$ is estimated by the signal to distance propagation model based on our prior work [14], and $s_{i j}^{L_{p}}$ is the observed RSS of the $L_{p}$ th dimension in landmark $i$ for $j=1 \ldots m$ locations.

Residual. The residual is represented as an error during the intermediate step of the localization process, which affects the final localization accuracy. Due to different localization principles, various algorithms have different residuals that are the leading factors contributing to the localization error. We next present our definition of residual for each algorithm together with the algorithm description in the following.

\section{Localization Algorithms}

1) Lateration Based: Localization using the lateration based approach is popular [5], [7], [10] and involves 2 steps: ranging and lateration.

Ranging Step: The purpose of the ranging step is to estimate the distance $d_{i}$ from the position of the targeting device $P=(x, y)$ to the $i$ th landmark $L_{i}=\left(x_{i}, y_{i}\right)$, which is represented as $d_{i}=\sqrt{\left(x_{i}-x\right)^{2}+\left(y_{i}-y\right)^{2}}$. A variety of physical modalities can be used to perform the ranging step such as Received Signal Strength (RSS), Time Of Arrival (TOA), Time Difference Of Arrival (TDOA) and hop count. In this work, we employ RSS to perform ranging. In particular, we used the linear regression approach in our prior work [14] to obtain the estimated distance $\hat{d}_{i}$.

Lateration Step: There are two popular methods, Nonlinear Least Squares(NLS) and Linear Least Squares $(L L S)$, to get the estimated position of the target device $\hat{P}=(\hat{x}, \hat{y})$. In NLS, from the estimated distance $\hat{d}_{i}$ and known positions $L_{i}=\left(x_{i}, y_{i}\right)$ of the landmarks, the position $(x, y)$ of the target device can be estimated by finding $(\hat{x}, \hat{y})$ satisfying:

$$
(\hat{x}, \hat{y})=\operatorname{argmin}_{x, y} \sum_{i=1}^{n}\left[\sqrt{\left(x_{i}-x\right)^{2}+\left(y_{i}-y\right)^{2}}-\hat{d}_{i}\right]^{2},
$$

where $i=1 \ldots n$ for $n$ total number of landmarks. NLS usually requires high computational complexity and is difficult to analyze.

NLS can be approximated by solving a set of linear equations [3] as $A \hat{P}=b$, where:

$$
A=\left(\begin{array}{cc}
x_{1}-\frac{1}{n} \sum_{i=1}^{n} x_{i} & x_{n}-\frac{1}{n} \sum_{i=1}^{n} x_{i} \\
\vdots & \vdots \\
y_{1}-\frac{1}{n} \sum_{i=1}^{n} y_{i} & y_{n}-\frac{1}{n} \sum_{i=1}^{n} y_{i}
\end{array}\right)
$$

and

$$
b=\frac{1}{2}\left(\begin{array}{c}
\left(x_{1}^{2}-\frac{1}{n} \sum_{i=1}^{n} x_{i}^{2}\right)+\left(y_{1}^{2}-\frac{1}{n} \sum_{i=1}^{n} y_{i}^{2}\right) \\
-\left(\hat{d}_{1}^{2}-\frac{1}{n} \sum_{i=1}^{n} \hat{d}_{i}^{2}\right) \\
\vdots \\
\left(x_{n}^{2}-\frac{1}{n} \sum_{i=1}^{n} x_{i}^{2}\right)+\left(y_{n}^{2}-\frac{1}{n} \sum_{i=1}^{n} y_{i}^{2}\right) \\
-\left(\hat{d}_{n}^{2}-\frac{1}{n} \sum_{i=1}^{n} \hat{d}_{i}^{2}\right) .
\end{array}\right)
$$

$A$ is described by the coordinates of landmarks, and $b$ is composed of estimated distances to the landmarks and the coordinates of landmarks. We call the above formulation of the problem linear Least Squares(LLS). The position estimation of the targeting device is done by solving $\hat{P}=\left(A^{T} A\right)^{-1} A^{T} b$.

Residual. We define the residual $R$ of the lateration method as the averaged difference between the estimated distance to each landmark $\hat{d}_{i}$ and the distance of the localized position to each landmark $\left\|\hat{P}-L_{i}\right\|$. The residual is represented as

$$
R=\frac{1}{n} \sum_{i=1}^{n}\left(\hat{d}_{i}-\left\|\hat{P}-L_{i}\right\|\right)^{2} .
$$

Intuitively, we expect the smaller residual can lead to better localization accuracy. We next provide an analysis of the relationship between the residual and the localization error.

Error Analysis. The residual can be represented in the following format by replacing $\hat{P}=\left(A^{T} A\right)^{-1} A^{T} b$ in Equation (5):

$$
R=\frac{1}{n} \sum_{i=1}^{n}\left(\hat{d}_{i}-\left\|\left(A^{T} A\right)^{-1} A^{T} b-L_{i}\right\|\right)^{2} .
$$

We can write $\hat{d}_{i}=d_{i}+\epsilon_{i}$ where $\epsilon_{i}$ is the distance estimation error, and substitute $\hat{d}_{i}$ in Equation (4). Then 
we have:

$$
\begin{gathered}
b=\frac{1}{2}\left(\begin{array}{c}
\left(x_{1}^{2}-\frac{1}{n} \sum_{i=1}^{n} x_{i}^{2}\right)+\left(y_{1}^{2}-\frac{1}{n} \sum_{i=1}^{n} y_{i}^{2}\right) \\
-\left(\left(d_{1}+\epsilon_{1}\right)^{2}-\frac{1}{n} \sum_{i=1}^{n}\left(d_{i}+\epsilon_{i}\right)^{2}\right) \\
\vdots \\
\vdots \\
\left(x_{n}^{2}-\frac{1}{n} \sum_{i=1}^{n} x_{i}^{2}\right)+\left(y_{n}^{2}-\frac{1}{n} \sum_{i=1}^{n} y_{i}^{2}\right) \\
-\left(\left(d_{n}+\epsilon_{n}\right)^{2}-\frac{1}{n} \sum_{i=1}^{n}\left(d_{i}+\epsilon_{i}\right)^{2}\right)
\end{array}\right) \\
=\frac{1}{2}\left(\begin{array}{c}
\left.x_{1}^{2}-\frac{1}{n} \sum_{i=1}^{n} x_{i}^{2}\right)+\left(y_{1}^{2}-\frac{1}{2} \sum_{i=1}^{n} y_{i}^{2}\right) \\
-\left(d_{1}^{2}+\epsilon_{1}^{2}+2 d_{1} \epsilon_{1}-\frac{1}{n} \sum_{i=1}^{n}\left(d_{i}^{2}+\epsilon_{i}^{2}+2 d_{i} \epsilon_{i}\right)\right) \\
\vdots \\
\left(x_{n}^{2}-\frac{1}{n} \sum_{i=1}^{n} x_{i}^{2}\right)+\left(y_{n}^{2}-\frac{1}{n} \sum_{i=1}^{n} y_{i}^{2}\right) \\
-\left(d_{n}^{2}+\epsilon_{n}^{2}+2 d_{n} \epsilon_{n}-\frac{1}{n} \sum_{i=1}^{n}\left(d_{i}^{2}+\epsilon_{i}+2 d_{i} \epsilon_{i}\right)\right)
\end{array}\right)
\end{gathered}
$$

we further define $\tilde{b}$ as:

$$
\tilde{b}=\frac{1}{2}\left(\begin{array}{c}
\left(x_{1}^{2}-\frac{1}{n} \sum_{i=1}^{n} x_{i}^{2}\right)+\left(y_{1}^{2}-\frac{1}{n} \sum_{i=1}^{n} y_{i}^{2}\right) \\
-\left(d_{1}^{2}-\frac{1}{n} \sum_{i=1}^{n} d_{i}^{2}\right) \\
\vdots \\
\left(x_{n}^{2}-\frac{1}{n} \sum_{i=1}^{n} x_{i}^{2}\right)+\left(y_{n}^{2}-\frac{1}{n} \sum_{i=1}^{n} y_{i}^{2}\right) \\
-\left(d_{n}^{2}-\frac{1}{n} \sum_{i=1}^{n} d_{i}^{2}\right)
\end{array}\right)
$$

Thus, $b$ can be rewritten by $\tilde{b}$ as:

$$
b=\tilde{b}-\frac{1}{2}\left(\begin{array}{c}
\epsilon_{1}^{2}+2 d_{1} \epsilon_{1}-\frac{1}{n} \sum_{i=1}^{n}\left(\epsilon_{i}^{2}+2 d_{i} \epsilon_{i}\right) \\
\vdots \\
\epsilon_{n}^{2}+2 d_{n} \epsilon_{n}-\frac{1}{n} \sum_{i=1}^{n}\left(\epsilon_{i}^{2}+2 d_{i} \epsilon_{i}\right)
\end{array}\right)
$$

and the true position of the targeting device $P$ is $P=$ $\left(A^{T} A\right)^{-1} A^{T} b$. Next, we continue Equation (6) by using Equation (9) and obtain

$$
R=\frac{1}{n} \sum_{i=1}^{n}\left(d_{i}+\epsilon_{i}-\left\|P-L_{i}-\left(A^{T} A\right)^{-1} A^{T}\left(\begin{array}{c}
M_{1} \\
\vdots \\
M_{n}
\end{array}\right)\right\|\right)^{2},
$$

where $M_{t}=\frac{1}{2}\left(2 d_{t} \epsilon_{t}+\epsilon_{t}^{2}-\frac{1}{n} \sum_{i=1}^{n}\left(2 d_{i} \epsilon_{i}+\epsilon_{i}^{2}\right)\right)$ with $t=1 \ldots n$. Since the quadratic terms of $\epsilon_{t}^{2}$ and $\epsilon_{i}^{2}$ are much smaller than the first degree terms of $\epsilon_{t}$ and $\epsilon_{i}$. We can leave out the quadratic terms of $\epsilon_{t}^{2}$ and $\epsilon_{i}^{2}$ and use $M_{t}^{\prime}=d_{t} \epsilon_{t}-\frac{1}{n} \sum_{i=1}^{n}\left(d_{i} \epsilon_{i}\right)$ with $t=1 \ldots n$ for residual $R$. Continuing Equation (10), the residual can be approximated as:

$$
\begin{aligned}
R & \approx \frac{1}{n} \sum_{i=1}^{n}\left(d_{i}+\epsilon_{i}-\left\|P-L_{i}-\left(A^{T} A\right)^{-1} A^{T}\left(\begin{array}{c}
M_{1}^{\prime} \\
\vdots \\
M_{n}^{\prime}
\end{array}\right)\right\|\right)^{2} \\
& =\frac{1}{n} \sum_{i=1}^{n}\left(d_{i}+\epsilon_{i}-\left\|P-L_{i}+\omega \epsilon\right\|\right)^{2}
\end{aligned}
$$

where $\epsilon$ is the vector of $\epsilon_{i}$, which is $\epsilon=\left(\epsilon_{1} \ldots \epsilon_{n}\right)^{T}$, and $\omega$ is given by,

$$
\begin{aligned}
\omega & =-\left(A^{T} A\right)^{-1} A^{T} \operatorname{Diag}\left\{d_{1} \cdots d_{n}\right\} \\
& +\frac{1}{n}\left(A^{T} A\right)^{-1} A^{T}\left(\begin{array}{c}
1 \\
\vdots \\
1
\end{array}\right)\left(\begin{array}{lll}
d_{1} & \cdots & d_{n}
\end{array}\right) \\
& =\left(A^{T} A\right)^{-1} A^{T}\left(\frac{1}{n}\left(\begin{array}{c}
1 \\
\vdots \\
1
\end{array}\right)\left(\begin{array}{lll}
d_{1} & \cdots & d_{n}
\end{array}\right)\right. \\
& \left.-\operatorname{Diag}\left\{d_{1} \cdots d_{n}\right\}\right) .
\end{aligned}
$$

Further, continuing Equation (11)

$$
\begin{aligned}
R & =\frac{1}{n} \sum_{i=1}^{n}\left(d_{i}+\epsilon_{i}\right. \\
& \left.-\sqrt{\left(P-L_{i}\right)^{T}\left(P-L_{i}\right)+2\left(P-L_{i}\right)^{T} \omega \epsilon+\epsilon^{T} \omega^{T} \omega \epsilon}\right)^{2} \\
& =\frac{1}{n} \sum_{i=1}^{n}\left(d_{i}+\epsilon_{i}-\sqrt{d_{i}^{2}+2\left(P-L_{i}\right)^{T} \omega \epsilon+\epsilon^{T} \omega^{T} \omega \epsilon}\right)^{2} .
\end{aligned}
$$

From our empirical study, we found that $\left(P-L_{i}\right)^{T} \omega \epsilon \approx$ $d_{i} \sqrt{\epsilon^{T} \omega^{T} \omega \epsilon}$, so $R$ can be further approximated as:

$$
\begin{aligned}
R & \approx \frac{1}{n} \sum_{i=1}^{n}\left(d_{i}+\epsilon_{i}-\left(d_{i}+\sqrt{\epsilon^{T} \omega^{T} \omega \epsilon}\right)\right)^{2} \\
& =\frac{1}{n} \sum_{i=1}^{n}\left(d_{i}+\epsilon_{i}-\left(d_{i}+\sqrt{\epsilon^{T} W \epsilon}\right)\right)^{2} \\
& =\frac{1}{n} \sum_{i=1}^{n}\left(\epsilon_{i}-\sqrt{\epsilon^{T} W \epsilon}\right)^{2},
\end{aligned}
$$

where $W$ is defined as $W=\omega^{T} \omega$. Therefore, the expectation value of the residual $R$ from Equation (14) can be obtained as:

$$
\begin{aligned}
E(R) & =E\left(\frac{1}{n} \sum_{i=1}^{n}\left(\epsilon_{i}-\sqrt{\epsilon^{T} W \epsilon}\right)\right)^{2} \\
& =E\left(\frac{1}{n} \sum_{i=1}^{n}\left(\epsilon_{i}^{2}-2 \sqrt{\epsilon_{i} \epsilon^{T} W \epsilon \epsilon_{i}}+\epsilon^{T} W \epsilon\right)\right) .
\end{aligned}
$$

We further define $\sigma_{i}$ to be the standard deviation of the estimated error $\epsilon_{i}$ to the $i$ th landmark. By further expanding Equation (15), we got

$$
\begin{aligned}
E(R)= & \frac{1}{n} E\left(\sum_{i=1}^{n} \epsilon_{i}^{2}\right)-\frac{2}{n} E\left(\sum_{i=1}^{n} \sqrt{\epsilon_{i} \epsilon^{T} W \epsilon \epsilon_{i}}\right) \\
& +E\left(\epsilon^{T} W \epsilon\right) \\
= & \frac{1}{n} \sum_{i=1}^{n} \sigma_{i}^{2}-\frac{2}{n} \sum_{i=1}^{n} W_{i, i} \sigma_{i}^{2}+\sum_{i=1}^{n} W_{i, i} \sigma_{i}^{2} \\
= & \frac{1}{n} \sum_{i=1}^{n} \sigma_{i}^{2}+\frac{n-2}{n} \sum_{i=1}^{n} W_{i, i} \sigma_{i}^{2} .
\end{aligned}
$$


We next turn to examine the localization error $e$ as the following:

$$
e=\|\hat{P}-P\|^{2}=\left\|\left(A^{T} A\right)^{-1} A^{T} b-P\right\|^{2} .
$$

Then, by substituting $b$ from Equation (4) and $P=$ $\left(A^{T} A\right)^{-1} A^{T} \tilde{b}$, we obtain

$$
\begin{aligned}
e & =\left\|P-P-\left(A^{T} A\right)^{-1} A^{T}\left(\begin{array}{c}
M_{1}^{\prime} \\
\vdots \\
M_{n}^{\prime}
\end{array}\right)\right\|^{2} \\
& =\left\|\left(A^{T} A\right)^{-1} A^{T}\left(\begin{array}{c}
M_{1}^{\prime} \\
\vdots \\
M_{n}^{\prime}
\end{array}\right)\right\|^{2} \\
& =\|\omega \epsilon\|^{2} \\
& =\epsilon^{T} \omega^{T} \omega \epsilon=\epsilon^{T} W \epsilon .
\end{aligned}
$$

We note that the derivation of Equation (18) is similar to Equation (11), and $\omega$ is given by Equation (12). Further, since the representation of $e$ in Equation (18) is the same as the third term in Equation (16) for the expectation value of the residual, we can thus write the expectation of the localization error $e$ as:

$$
E(e)=\sum_{i=1}^{n} W_{i, i} \sigma_{i}^{2} .
$$

We note that $W$ is a symmetric matrix. It is thus nonnegative definite and $W_{i, i}$ is equal or larger than zero.

Based on the above error analysis, we observed that both the residual and localization error are highly correlated with distance estimation error, which is derived from the RSS measurement in our study. The noise, measurement errors, and system bias in the RSS measurement will impact the distance estimation accuracy, and consequently the residual and localization error. Moreover, we found that larger residual may cause larger localization error, which suggests improving localization accuracy through selecting smaller residual. In this study, we derive methods to show that how to select from the combination of wireless devices' multiple frequencies and multiple power levels so that to reduce the residual and the corresponding localization error.

2) Fingerprint Matching: The Radar algorithm [1] is a classic machine learning method based on fingerprint matching, which requires building a signal map consisting of RSS fingerprints with known $(x, y)$ locations. On top of the basic fingerprints in RADAR, Gridded Radar (GR) uses the IMG (Interpolated Map Grid) to build an interpolated signal map. GR builds a regular grid of tiles over the localization area that describes the expected fingerprint for the area described by each tile. Given a RSS fingerprint of a targeting device, GR returns the position $(x, y)$ of the closest fingerprint in the interpolated signal map to the one of the targeting device as the location estimation, where closest means the Euclidean distance of the fingerprints in the signal space.

Residual: Given the observed fingerprint of a targeting device at location $j$, the residual of Gridded Radar is defined as the Euclidean distance of the fingerprint from the targeting device to the interpolated signal map in signal space:

$$
R=\sqrt{\sum_{i=1}^{n} \sum_{\text {col=1 }}^{\operatorname{col} M} \sum_{\text {row }=1}^{\text {row } M} \| s_{i j}^{L_{p}}-S G_{i}^{L_{p}}(\text { row }, \text { col }) \|^{2}}
$$

where $n$ is the number of landmarks, $s_{i j}^{L_{p}}$ is the RSS reading of the targeting device at location $j$ in the $L_{p}$ th dimension of the $i$ th landmark where $L_{p}=L_{1} \ldots L_{q}$ with $q$ as the total number of dimensions under that landmark, and $S G_{i}^{L_{p}}$ represents the interpolated RSS matrix of the $L_{p}$ th dimension for the $i$ th landmark, where row is the total number of rows in the interpolated RSS matrix with row $=1 \ldots$ row $M$ and col is the total number of columns in the interpolated RSS matrix with $\operatorname{col}=1 \ldots \operatorname{col} M$. We note that for different landmark $i$, dimension $L_{p}$ can be different.

3) Probabilistic Based: In this work, we choose to study Area Based Probability (ABP) [4], which is a representative method based on the statistical Bayes' Rule to perform location estimation. ABP also uses the interpolated signal map as in GR and computes the likelihood of the observed fingerprint of a targeting device matching a fingerprint of each tile in the interpolated signal map. It returns the top probability tile set whose sum matches the desired confidence level $\alpha$. In particular, by using Bayes' rule, ABP computes the probability of being at each tile $T_{j}$ on the floor given the fingerprint of the targeting device $S_{T}$ :

$$
P\left(T_{j} \mid S_{T}\right)=\frac{P\left(S_{T} \mid T_{j}\right) \times P\left(T_{j}\right)}{P\left(S_{T}\right)}
$$

Residual: Since the number of tiles in the returned tile set represents how well the algorithm captures the true location of the targeting device, the less the number of returned tiles indicates the better the probabilistic matching is found. We thus define the residual of ABP as the number of tiles returned based on the confidence level $\alpha$.

4) Bayesian Networks: Bayesian Networks [4] utilizes the Bayesian Graphical Model to compute the distribution of the position $(x, y)$ of a targeting device. In particular, Bayesian Networks encodes the relationship between the RSS readings and the location based on the signal-distance propagation model. The initial parameters of the model are unknown, and the training set collected from multiple known locations is used to adjust the parameters of the model according to the relationships encoded in the network. 


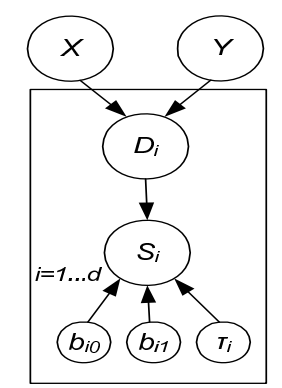

Figure 1. Bayesian graphical model in our study.

Figure 1 depicts the basic Bayesian Graphical Model The random variables $s_{i}, i=1 \ldots n$ denotes the expected signal strength of the corresponding dimension (e.g., a combination of frequency and power level) in the landmark $L_{i}$. The values of these random variables depend on the Euclidean distance $d_{i}$ between the landmark's location $\left(x_{i}, y_{i}\right)$, and the location where the signal $s_{i}$ is measured $(x, y)$. The baseline expected value of $s_{i}$ follows a signal propagation model $s_{i}=b_{0 i}+b_{1 i} \log \left(d_{i}\right)$, where $b_{0 i}, b_{1 i}$ are the parameters specific to each $L_{i}$. The distance $d_{i}=\sqrt{\left(x-x_{i}\right)^{2}+\left(y-y_{i}\right)^{2}}$ in return depends on the location $(x, y)$ of the measured signal. The network models noise and outliers by modeling the expected value, $s_{i}$, as a $t$-distribution around the above propagation model, with variance $\tau_{i}, s_{i} \sim t\left(b_{0 i}+\right.$ $\left.b_{1 i} \log d_{i}, \tau_{i}, 2\right)$. Using the training fingerprints $T$, the network can learn the specific values for all the unknown parameters $b_{0 i}, b_{1 i}, \tau_{i}$ and the joint distribution of $(x, y)$ location of the targeting device.

In general, there is no closed form solution for the returned joint distribution of the $(x, y)$ location. Therefore, we use a Markov Chain Monte Carlo (MCMC) simulation approach to draw samples from the joint density, and then pick the samples that give a 95\% confidence on the density. Finally, we approximate the returned area by the tiles where those samples fall.

Residual: When computing the joint distribution of the location of the targeting device, we define the residual as: $R=\sqrt{\sigma_{x}^{2}+\sigma_{y}^{2}}$, where $\sigma_{x}$ and $\sigma_{y}$ are the standard deviation of the position variable $x$ and $y$, which represents how stable the estimated position distribution is. Intuitively, the more stable the distribution of the estimated position is, we expect better localization accuracy.

\section{ExPERIMENTAL Methodology AND SELECTION STRATEGY}

In this section, we first describe our experimental methodology by presenting the testbed infrastructure and the data collection process. We then present our dimension selection strategies and selection procedures to characterize the impact of multiple frequencies and multiple power levels from the transmitting device.

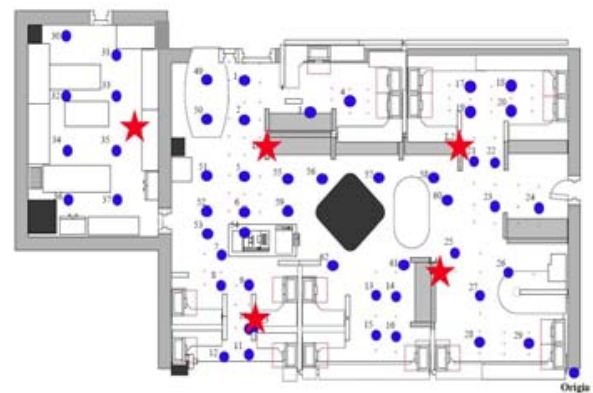

Figure 2. Burchard 2nd floor map

\section{A. Testbed Infrastructure and Data Collection}

RSS measurements were conducted using a localization testbed [2] with active RFID tags and readers from InPoint [16]. Each RFID tag has a unique identifier and periodically beacons its identifier, which is received by the landmarks. The tags transmit without collision avoidance or detection; the readers identify overlapping signals. We connect the RFID reader to a Linux machine with $2 \mathrm{GHz}$ CPU, 1GB RAM and 20GB disk to serve as our landmark. In our localization testbed, landmarks continuously monitor the channels' traffic at the packet-level and forward their observed RSS readings to a central server. The server is responsible for averaging the RSS readings over multiple packets to produce fingerprints. In our experiment, each averaged RSS reading is obtained over 100 packets.

We collected experimental data on the second floor of Buchard building at Stevens Institute of Technology, which is a $70 \mathrm{ft} \times 80 \mathrm{ft}$ area as shown in Figure 2. This is a large lab area containing office wall dividers and furniture, such as desks, shelves and chairs. We deployed 5 landmarks and collected RSS fingerprints for 51 testing points. Landmarks and testing positions are shown as stars and dots respectively. We programmed the RFID tag to transmit between two frequencies, $400 \mathrm{MHz}$ and $900 \mathrm{MHz}$, alternatively. Data collection is then repeated for 3 transmission power levels $(0 \mathrm{dBm}, 5 \mathrm{dBm}$, and $10 \mathrm{dBm})$ at 2 frequencies $(400 \mathrm{MHz}$ and $900 \mathrm{MHz})$ by placing three transmitters at the same testing location, which approximates a system with 6 different dimensions (power level and frequency combinations). Given the number of testing points and the dimension of each testing point, the total number of fingerprints in our experimental dataset is $51 \times 30=1530$.

We used the leave-one-out method for evaluating the localization performance under different combinations of multiple frequencies and multiple power levels, which means that we chose one location as the testing point, whereas the rest of the locations (i.e., 50 points) as the training data.

\section{B. Synthetic Data Generation}

To study the propagation of the RSS readings and validate our approach, we also generated a synthetic RSS data set using a simple signal propagation model [14] 
with Gaussian noise. To be comparable with the real data, we use the same setup including testing area, landmarks and testing points' positions as described in Section IV-A.

The synthetic data set is generated in such a way that it mimics the real data set in terms of the fitting to the path loss signal propagation model. Specifically, we used the $R^{2}$ metric (also called the coefficient of determination) and made the resulting values of $R^{2}$ from the synthetic data as close to those from the real data as possible. We note that the $R^{2}$ value varies from 0 to 1 , with 1 indicating a perfect fit and a value close to 0 indicating a bad fit.

\section{Performance Baseline}

Based on our data collection using multiple frequencies (i.e., $400 \mathrm{MHz}$ and $900 \mathrm{MHz}$ ) and multiple power levels (i.e., $0 \mathrm{dBm}, 5 \mathrm{dBm}$, and $10 \mathrm{dBm}$ ), we have total 6 single dimension RSS readings for each landmark. We can perform localization by using each individual dimension, which has a fixed frequency and a fixed power level (e.g., $900 \mathrm{MHz}$ and $0 \mathrm{dBm}$ ). We use the localization performance from running these 6 single dimension data sets to report baseline results, which will be used to compare with those from different combination of frequency and power level through selection.

\section{Selection Strategies}

We next develop three selection methods, namely Whole Dimension, Matching Characteristic, and Constrained Landmark, which explore which utilize different combination of multiple frequencies and multiple power levels to perform localization.

Whole Dimension. This is a straightforward selection method. Each combination of a frequency and a power level from an individual landmark is treated as one dimension. Given there are 5 landmarks and 6 possible combinations from each landmark in our experimental setup, there are total 30 dimensions. In this selection method, all the dimensions are used to construct a fingerprint. For instance, $S=$ $\left[s_{1}^{1}, \ldots, s_{1}^{6}, \ldots, s_{i}^{1}, \ldots, s_{i}^{6}, \ldots s_{5}^{1}, \ldots, s_{5}^{6}\right]$ is the fingerprint for a testing point when the Whole Dimension selection method is used. This method may incur high computational cost for certain algorithms such as $\mathrm{BN}$ since it needs to compute the joint position distribution across high dimensions.

Matching Characteristic. In our experiments, each dimension has a fixed frequency and power level, which we call a signal characteristic. In contrary to the Whole Dimension method, the Matching Characteristic method tries to keep the number of dimensions as the same as the number of landmarks when selecting RSS fingerprints. In the Matching Characteristic selection method, we request the same characteristic to be chosen from the 6 dimensions for each landmark, that is, during one selection process for localization, the combination of the frequency and power level selected should be the same across all landmarks. Based on this selection metric, there are total 6 possible combinations of frequency and power level. To perform localization, Matching Characteristic will use our selection metrics to determine what is the appropriate combination of the frequency and power level to be chosen as the characteristic for each testing point in our experiments.

When Matching Characteristic selection is applied using RMSE, RMSE will be calculated for each combination of the frequency and power level and compare across all the 6 possible combinations. Within each combination, we can first calculate $R M S E_{i}^{L_{p}}(\hat{S})$ for dimension $L_{p}$ of landmark $i$ based on Equation (1). We then calculate the RMSE for this combination over all the landmarks as $R M S E_{L_{p}}(\hat{S})=\sqrt{\sum_{i=1}^{n} R M S E_{i}^{L_{p}}(\hat{S})^{2}}$ with $L_{p}=1 \ldots 6$. The RSS fingerprints from the frequency and power level combination that produces the minimum value of RMSE $R M S E_{\text {min }}=\min _{L_{p}}\left(R M S E_{L_{p}}(\hat{S})\right)$ over all the combinations will be selected as the appropriate characteristic for performing localization.

On the other hand, When Matching Characteristic selection is applied using residual, the residual is calculated for all the frequency and power level combinations. We choose the frequency and power level combination that produces the minimum residual as characteristic to perform localization for each algorithm. For different testing points, the selected characteristic may be different, because it is possible that different testing points have different combinations of frequency and power level that produces the minimum residual.

Constrained Landmark. Instead of using the full dimension of the data, the objective of the Constrained Landmark takes advantage of using multiple frequencies and multiple power levels, but without increasing the dimension for algorithm computation and without fixing the characteristics across landmarks. 'Constrained' means that the RSS fingerprint must be constructed by selecting from different landmarks. However, the dimension chosen in each landmark could be different from different landmarks. Particularly, the constructed RSS fingerprint is composed of element $S_{i}^{L_{p}}$ with $i=$ $1 \ldots 5$ in our experiments, and for each landmark $i$, the dimension $L_{p}$ can be selected differently corresponding to different combinations of frequency and power level. Therefore, there can be total $6^{5}=7776$ different combinations when applying Constrained Landmark selection in our experiments. The computational cost of using Constrained Landmark is higher than using Matching Characteristic.

This selection method will be used together with our selection metrics to perform localization by each algorithm. When the Constrained Landmark selection method is applied by using RMSE, the same calculation of RMSE will be performed as described in the Matching 


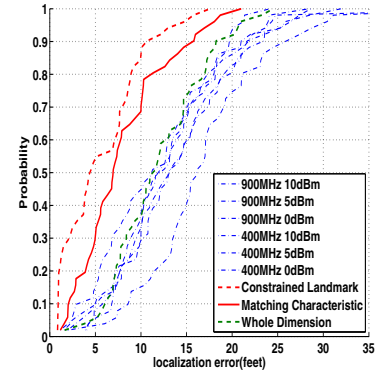

(a) LLS, Residual based selection

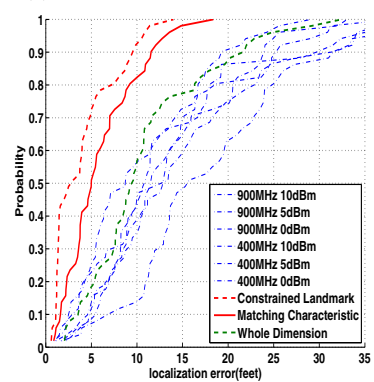

(c) NLS, Residual based selection

Figure 3. Lateration based algorithr under different selection strategies.

Characteristic selection to search for the frequency and power level combination with the minimum RMSE, however, there are total $6^{5}$ combinations. Likewise, when the Constrained Landmark selection method is applied by using residual, the same computation of residual will be performed as presented in Matching Characteristic. The combination of the frequency and power level that produces the minimum residual across the $6^{5}$ combinations will be selected.

\section{Evaluation Results}

In this section, we experimentally evaluate the impact of multiple frequencies and multiple power levels on the performance of localization algorithms. We study all the algorithms described in Section III-C and evaluation is conducted using both selection metrics (RMSE and Residual) and all three selection strategies (Whole Dimension, Constrained Landmark, and Matching Characteristic). In addition, we study the feasibility of using synthetic data for our multi-dimension evaluation.

In all our studies, localization performance is characterized by the Accuracy metric, which is the Euclidean distance between the estimated location from the algorithm and the device's actual location.

\section{A. Lateration Based Algorithms}

Figure 3 presents the localization accuracy Cumulative Distributed Function (CDF) for lateration based algorithms under different selection strategies. Results are included for both LLS and NLS algorithms, and for both residual and RMSE based selection metrics. Performance for each single dimension is also plotted as our baseline for comparison.

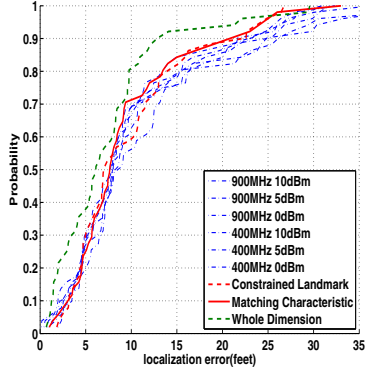

(a) Residual based selection

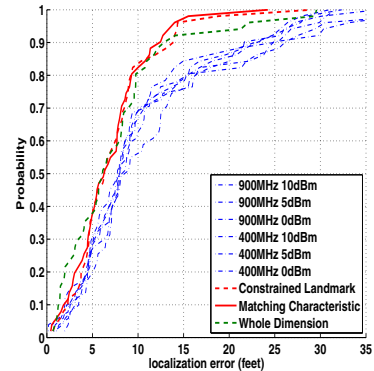

(b) RMSE based selection
Figure 4. Fingerprint Matching algorithm (GR): Localization accuracy $\mathrm{CDF}$ under different selection strategies.

Firstly, we notice that the accuracy CDFs from all 6 single dimensions are similar to each other. This indicates that simply changing the dimension characteristics, for example from using one transmitting frequency to another, is unlikely to offer much accuracy improvements.

Secondly, contrary to our initial expectations, although using Whole Dimension achieves a performance close to the best among all the single dimensions, the additional information does not bring significant accuracy improvements for lateration based algorithms. This actually is directly related to how this set of algorithms is designed. For lateration based algorithms, path loss signal propagation model is strictly enforced for distance estimation without any accommodation for noise in the data. Noisy RSS information thus directly translates to uncertainty in the lateration phase. Furthermore, full dimension only provides additional sets of distance estimation to the same group of landmarks. Thus, in this selection approach more uncertainty is added to lateration along with more distance information, but with no further geometrical constraints. Simple least squares metric is not sufficient to cope with the additional uncertainty.

Finally, compliant to our theoretical analysis, the intelligent selection strategies based on both metrics successfully achieve better localization accuracy for lateration based algorithms. For example, using Constrained Landmark with residual metric, LLS's median error decreases from $12 \mathrm{ft}$ to $5 \mathrm{ft}$ (58\% improvement) and the 90th percentile error changes from $22 \mathrm{ft}$ to $10 \mathrm{ft}$ (54\% improvement). Similarly, using Matching Characteristics with RMSE metric, LLS's median error decreases from $12 \mathrm{ft}$ to $7 \mathrm{ft}$ ( $42 \%$ improvement) and the 90th percentile error changes from $22 \mathrm{ft}$ to $15 \mathrm{ft}$ (32\% improvement). We also notice that Constrained Landmark significantly outperforms Matching Characteristics and always performs the best among all the selection strategies. Such accuracy gain however, as pointed in Section IV, comes with significantly more computational cost.

In summary, for lateration based algorithms, it is not sufficient to simply deploy multi-frequency and multipower levels and expect performance improvements. Intelligent selection strategies are required to prune the RSS measurements to better utilize the additional 


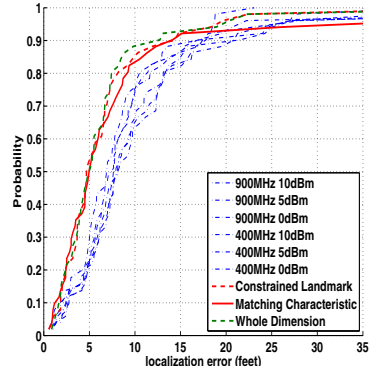

(a) Residual based selection

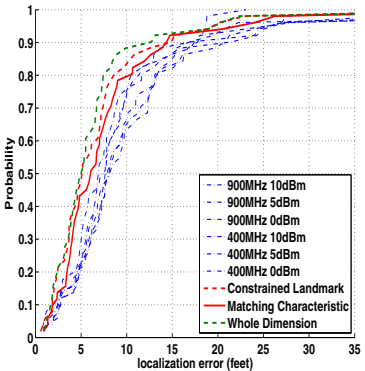

(b) RMSE based selection
Figure 5. Probabilistic based algorithm (ABP): Localization accuracy CDF under different selection strategies.

dimensions.

\section{B. Fingerprint Matching Algorithm (GR)}

Figure 4 shows the localization accuracy CDFs of Gridded Radar algorithm while using residual and RMSE as metric under different selection strategies. We again observe that performance for single dimension consistently agrees with each other, indicating no improvement by simply changing transmission power or frequency. Whole Dimension, however, outperforms single dimensions. Specifically, the median error decreases from 8 $\mathrm{ft}$ to $6 \mathrm{ft}$ (20\% improvement) and the 90th percentile error shrinks from $25 \mathrm{ft}$ to $13 \mathrm{ft}$ (48\% improvement). This is because GR is a machine learning based matching algorithm which straightforwardly benefits from a more precise signal map generated by adding more dimensions.

Furthermore, the intelligent selection strategies, Constrained Landmark and Matching Characteristic, tend to improve the performance over single dimensions. Although they do not necessarily offer much more accuracy advantage over using full dimension directly, the lower computational cost, especially for Matching Characteristic, compared to Whole Dimension makes them attractive for achieving better localization than using single dimensions only. We also noticed that RMSE based selection strategies perform better than residual based selection strategies. Overall, for GR algorithm, deploying multifrequency and multi-power can bring around significant performance improvements. There is also a tradeoff between localization accuracy and computational cost when deciding on different selection strategies.

\section{Probabilistic Based Algorithm (ABP)}

Figure 5 compares the effect of different selection metrics and selection strategies on ABP algorithm. We notice very similar overall results to the GR algorithm we just described in Section V-B. This is because GR and ABP are very similar in nature. They both localize with a sampled signal map: GR uses Euclidean distance in signal space while ABP uses a probabilistic method to compare the similarity of RSS fingerprints. Therefore, for ABP algorithm, employing multiple frequencies and

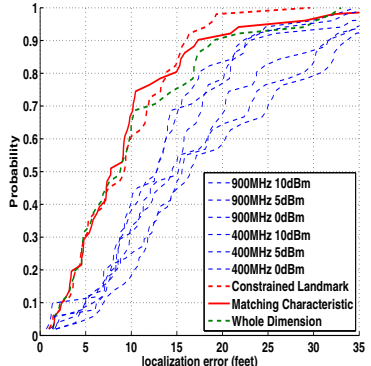

(a) Residual based selection

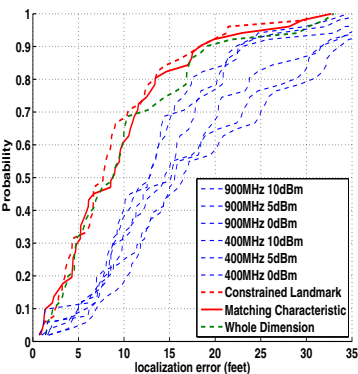

(b) RMSE based selection Figure 6. Bayesian Networks algorithm: Localization accuracy CDF under different selection strategies.

power levels and a simple Whole Dimension strategy are most effective in improving localization accuracy.

\section{Bayesian Networks Algorithm}

Results for selection strategies comparison on $\mathrm{BN}$ algorithm are presented in Figure 6. We see that all three selection approaches improve the accuracy in a similar way. Comparatively, $\mathrm{BN}$ is able to handle full dimension more gracefully than LS algorithms. This is because although both algorithms rely on the data's compliance with path loss signal propagation model, for $\mathrm{BN}$ noise in RSS is inherently incorporated in the algorithm model design. Such direct incorporation of noise consideration allows it to take better advantage of the additional dimensions than LS algorithms. We also observe a similar accuracy improvement to GR and ABP. For instance, when using residual metric, all the selection approaches can reduce the median error from $13 \mathrm{ft}$ to $9 \mathrm{ft}$ (30\% improvement) and the 90th percentile error from $28 \mathrm{ft}$ to $18 \mathrm{ft}$ ( $36 \%$ improvement). This is on similar magnitude with the $20 \%$ and $48 \%$ improvement offered by GR.

\section{E. Discussion on usage of synthetic data}

Synthetic data is often used in localization studies because of the difficulty measuring RSS in real environments. In this section, we thus evaluate the effectiveness of synthetic data in our multi-frequency and multi-power study. Specifically, we investigated whether adjusting the Gaussian noise in our signal propagation model sufficient characterizes the algorithms' responses.

The synthetic data generation is described in Section IV-B and the result of our study using the corresponding synthetic data is presented in Figure 7. At a qualitative level, we see that the synthetic data is not able to produce similar responses as actual RSS data. For example, it does not capture the significant accuracy advantage for using intelligent selection approaches over Whole Dimension for lateration based algorithms. We thus conclude that simple approach of path loss signal propagation model with random Gaussian noise for synthetic data generation is not sufficient to characterize the effects of using multiple frequencies and multiple power levels. 


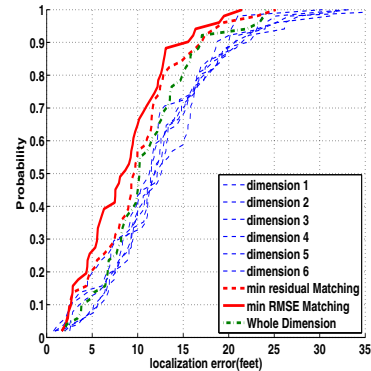

(a) LLS

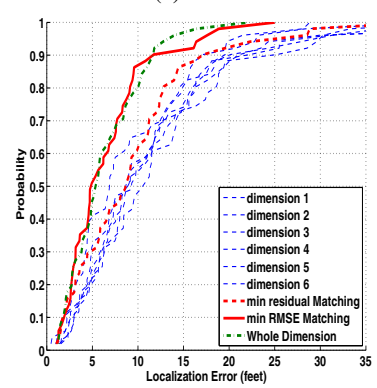

(c) GR

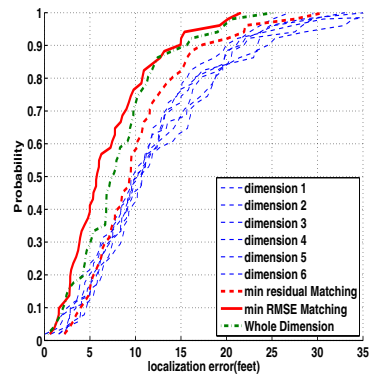

(b) NLS

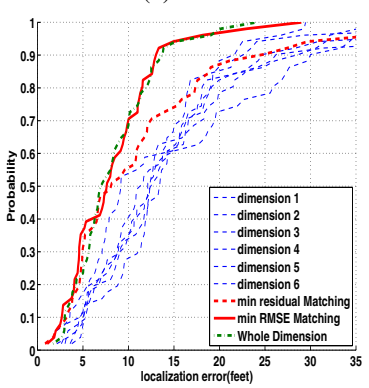

(d) ABP
Figure 7. Synthetic data: localization accuracy CDF under different selection strategies over all algorithms.

\section{CONCLUSION}

In this paper, we explored improving wireless localization performance in face of multipath effects by utilizing multiple frequencies and transmission powers. We studied a broad range of algorithms employing received signal strength (RSS) to perform localization including lateration based, fingerprint matching, probabilistic based, and Bayesian Networks. We developed three selection strategies, Whole Dimension, Matching Characteristic, and Constrained Landmark. Our selection strategies work with selection metrics, e.g., deviation of RSS readings and residual, to form high quality RSS fingerprints out of multiple dimensions resulting from the use of multi-frequency and multi-power.

We performed both a theoretical analysis as well as experimental validation of our approach. Our analysis on the Linear Least Squares (LLS) algorithm showed that selection should improve the localization performance. Further, our trace-driven study on a localization testbed with RFID transmitters varying the frequency and power simultaneously showed that using multiple frequencies and power levels improved the performance of all the algorithms under study. We also found that different algorithms react differently to the selection strategies. Lateration based algorithms were the most sensitive to selection, whereas algorithms rooted in machine learning were much more robust to adding dimensions. In addition, we observed there is a tradeoff between improving performance and reducing computational cost through intelligent selection.

\section{REFERENCES}

[1] P. Bahl and V. N. Padmanabhan. RADAR: An in-building RFbased user location and tracking system. In Proceedings of the IEEE International Conference on Computer Communications (INFOCOM), pages 775-784, March 2000.

[2] Y. Chen, G. Chandrasekaran, E. Elnahrawy, J. Francisco, K. Kleisouris, X. Li, R. P. Martin, R. S. Moore, and B. Turgut. GRAIL: A general purpose localization system. Sensor Review, special edition, Localisation Systems, , 28(2):115-124, 2008.

[3] Y. Chen, J. Francisco, W. Trappe, and R. P. Martin. A practical approach to landmark deployment for indoor localization. In Proceedings of the Third Annual IEEE Communications Society Conference on Sensor, Mesh and Ad Hoc Communications and Networks (SECON), September 2006.

[4] E. Elnahrawy, X. Li, and R. P. Martin. The limits of localization using signal strength: A comparative study. In Proceedings of the First IEEE International Conference on Sensor and Ad hoc Communcations and Networks (SECON 2004), pages 406-414, Oct. 2004.

[5] P. Enge and P. Misra. Global Positioning System: Signals, Measurements and Performance. Ganga-Jamuna Pr, 2001.

[6] K. Kleisouris, Y. Chen, J. Yang, and R. P. Martin. The impact of using multiple antennas on wireless localization. In Proceedings of the Fifth Annual IEEE Communications Society Conference on Sensor, Mesh and Ad Hoc Communications and Networks (SECON), June 2008.

[7] K. Langendoen and N. Reijers. Distributed localization in wireless sensor networks: a quantitative comparison. Comput. Networks, 43(4):499-518, 2003.

[8] X. Li, Y. Zhang, and A. M.G. Multifrequency-based range estimation of rfid tags. In IEEE International Conference on RFID, Apr. 2009.

[9] D. Madigan, E. Elnahrawy, R. Martin, W. Ju, P. Krishnan, and A. S. Krishnakumar. Bayesian indoor positioning systems. In Proceedings of the IEEE International Conference on Computer Communications (INFOCOM), pages 324-331, March 2005.

[10] D. Niculescu and B. Nath. Ad hoc positioning system (APS). In Proceedings of the IEEE Global Telecommunications Conference (GLOBECOM), pages 2926-2931, 2001.

[11] N. Priyantha, A. Chakraborty, and H. Balakrishnan. The cricket location-support system. In Proceedings of the ACM International Conference on Mobile Computing and Networking (MobiCom), pages 32-43, Aug 2000.

[12] H. Ren and M. Q.-H. Meng. Power adaptive localization algorithm for wireless sensor networks using particle filter. IEEE Transactions on Vehicular Technology, 58(5), 2009.

[13] R. Want, A. Hopper, V. Falcao, and J. Gibbons. The active badge location system. ACM Transactions on Information Systems, 10(1):91-102, Jan. 1992.

[14] J. Yang and Y. Chen. Indoor localization using improved RSSbased lateration methods. In IEEE Global Communications Conference 2009 Wireless Networking Symposium (Globecom), Honolulu, Hawaii, USA, November 2009.

[15] S. Yang, J. Yi, and H. Cha. HCRL: A Hop-Count-Ratio based Localization in Wireless Sensor Networks. In Proceedings of the Fourth IEEE Communications Society Conference on Sensor, Mesh, and Ad Hoc Communications and Networks (SECON), June 2007.

[16] Y. Zhang, G. Bhanage, W. Trappe, Y. Zhang, and R. Howard. Facilitating an active transmit-only rfid system through receiverbased processing. In Proceedings of the Fourth Annual IEEE Communications Society Conference on Sensor, Mesh, and Ad Hoc Communications and Networks (SECON), 2007.

[17] Y. Zhang, X. Li, and A. M.G. Array processing for RFID tag localization exploiting multi-frequency signals. In SPIE Symposium on Defense, Security, and Sensing, Apr. 2009.

[18] Y. Zhang, Z. Li, and W. Trappe. Power-modulated challengeresponse schemes for verifying location claims. In Proceedings of IEEE Global Telecommunication Conference (Globecom), Nov. 2007.

[19] Y. Zhou, J. He, K. Chen, J. Chen, and A. Liang. An area localization scheme for large scale underwater wireless sensor networks. In Proceedings of International Conference on Communications and $M$ obile Computing, Jan. 2009. 